\title{
Peptides Obtained by Enzymatic Decomposition of Mackerel Prevent Nonalcoholic Steatohepatitis in Sprague-Dawley Rats fed a High-fat and High-cholesterol Diet
}

Katsuhisa Omagari ${ }^{1,2, *}$, Ayumi Fukuda ${ }^{1}$, Machiko Suga ${ }^{1}$, Ayumi Ogata ${ }^{1}$, Shinta Nishioka ${ }^{2}$, Kazuhito Suruga ${ }^{1,2}$, Mayuko Ichimura ${ }^{3}$, Koichi Tsuneyama ${ }^{3}$

1. Department of Nutrition, Faculty of Nursing and Nutrition, University of Nagasaki, 1-1-1 Manabino, Nagayo-cho, Nagasaki 851-2195 Japan; E-Mails: omagari@sun.ac.jp (K.O.); m3218001@sun.ac.jp (A.F.); b3214016@sun.ac.jp (M.S.); b3214002@sun.ac.jp (A.O.); suruga@sun.ac.jp (K.S.)

2. Division of Nutritional Science, Graduate School of Human Health Science, University of Nagasaki, 1-1-1 Manabino, Nagayo-cho, Nagasaki 851-2195 Japan; E-Mail: snishioka@zeshinkai.or.jp (S.N.)

3. Department of Pathology and Laboratory Medicine, Tokushima University Graduate School, 318-15, Kuramoto-cho, Tokushima 770-8503, Japan; E-Mails: ichimura.mayuko@tokushimau.ac.jp (M.I.); tsuneyama.koichi@tokushima-u.ac.jp (K.T.)

* Correspondence: Katsuhisa Omagari; E-Mail: omagari@sun.ac.jp; Tel. \& Fax: +81-95-813-5201

Academic Editor(s): Tatsuo Kanda

OBM Hepatology and Gastroenterology

2018 , volume 2 , issue 3

doi:10.21926/obm.hg.1803008
Received: April 9, 2018

Accepted: July 27, 2018

Published: August 4, 2018

\section{Abstract:}

Background: The pathogenesis of nonalcoholic steatohepatitis (NASH) is thought to be multifactorial, and oxidative stress may play an important role in the development of NASH. Peptides obtained by enzymatic decomposition of mackerel (EMP) including selenoneine were recently reported to possess antioxidative activity. Thus, EMP is a potential dietary strategy for the prevention of NASH.

Methods: Nine-week-old male Sprague-Dawley rats were fed a high-fat and high-cholesterol (HFC) diet with or without EMP (HFC supplemented with 1\%, $2.5 \%$, or $5 \%(w / w)$ EMP) for 9 weeks ( $n=6$ -

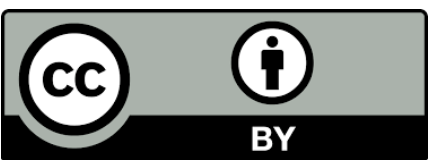

(C) 2018 by the author. This is an open access article distributed under the conditions of the Creative Commons by Attribution License, which permits unrestricted use, distribution, and reproduction in any medium or format, provided the original work is correctly cited. 
7/group). Histopathology and serology were evaluated as well as expressions of genes in the liver involved in fibrogenesis, inflammation, oxidative stress, and lipid metabolism.

Results: Histologically, the HFC diet with EMP tended to reduce NASH progression compared to the HFC diet without EMP (NASH diagnostic rate: $80 \%$ vs $100 \%$ ). Serum leptins in rats fed the HFC diet with EMP tended to be lower than those in rats fed an HFC diet without EMP. Immunohistochemically, the expression of 4-hydroxynonenal as a marker of oxidative stress in the liver tended to be less evident in rats fed the HFC diet with EMP than without EMP. MRNA levels of NF-KB tended to be lower in rats fed the HFC diet with EMP than without EMP, whereas mRNA levels of GPX-1 tended to be higher in rats fed the HFC diet with EMP than without EMP in an EMP-dose dependent manner. Histological findings and hepatic mRNA expressions indicate that the HFC diet containing $1 \%$ or $2.5 \%(\mathrm{w} / \mathrm{w})$ EMP was more likely to reveal a preventive effect against NASH progression than the HFC diet with $5 \%$ (w/w) EMP.

Conclusions: Our data suggests that EMP containing selenoneine may prevent NASH progression, presumably through its antioxidative activity. The optimal dose of EMP requires further study.

\section{Keywords}

Selenoneine; enzymatic decomposition of mackerel; nonalcoholic steatohepatitis; high-fat and high-cholesterol diet; antioxidative activity

\section{Introduction}

Nonalcoholic fatty liver disease (NAFLD) is currently recognized as the most common cause of chronic liver disease in many countries. NAFLD consists of two clinical entities: nonalcoholic fatty liver (NAFL), which is a mostly benign, nonprogressive disease and nonalcoholic steatohepatitis (NASH), which can progress to cirrhosis or even hepatocellular carcinoma. NASH is histologically characterized by hepatic steatosis associated with liver cell injury (ballooning degeneration) and inflammation [1]. The pathogenesis of NASH is thought to be multifactorial, and oxidative stress may play an important role in the development of NASH [2, 3]. Nutritional factors are commonly associated with $\mathrm{NASH}$; however, there are few fully proven treatments for $\mathrm{NASH}$, making it imperative to develop novel dietary strategies that can prevent NASH.

Selenium is an essential micronutrient for humans and animals, and seafood is one of the major sources of selenium in humans [4, 5]. The antioxidant function of selenium plays protective roles in various human diseases including prostate, lung, or colon cancers; immunodeficiency; and heart diseases [6]. Selenoneine is the major form of selenium found in aqueous extracts and is the major selenium compound in the muscles of fish such as tuna, mackerel, and swordfish [7, 8]. Selenoneine has strong antioxidant properties and a detoxifying function against methylmercury toxicity [8]. Recently, peptides obtained by enzymatic decomposition of mackerel (EMP) containing selenoneine were reported to possess antioxidative activity and affect cytokine secretion such as interleukin-10 (IL-10) $[9,10]$. Therefore, this EMP can be a potential dietary strategy for the prevention of NASH. 
The objective of this study was to evaluate the preventive effect of EMP against NASH progression in Sprague-Dawley (SD) rats that were fed a high-fat and high-cholesterol diet (HFC; NASH model) [11]. The mechanisms underlying the effects of EMP, specifically in relation to fibrogenesis, inflammation, oxidative stress, and cholesterol and lipid metabolism in the liver were also investigated.

\section{Materials and Methods}

All procedures performed on the animals were approved by the Animal Use Committee of the University of Nagasaki, and the animals were maintained in accordance with the University of Nagasaki's Guidelines for the Care and Use of Laboratory Animals.

\subsection{Animals and experimental design}

Eight-week-old male Sprague-Dawley (SD) rats were purchased from Japan SLC (Hamamatsu, Japan) and housed individually in a temperature- and humidity-controlled room $\left(22-24{ }^{\circ} \mathrm{C}\right.$ and 50 $60 \%$ relative humidity) with a 12 -hour light-dark cycle. After a one-week acclimation period with standard rodent chow (MF; Oriental Yeast, Tokyo, Japan) and water ad libitum, the rats were randomly divided into five groups that were fed for 9 weeks as follows: the control group $(n=6)$ was fed MF as the normal diet, the HFC group $(n=6)$ was fed a high-fat and high-cholesterol (HFC) diet, the EMP1\% group $(n=6)$ was fed a HFC diet supplemented with $1 \%(w / w)$ EMP, the EMP2.5\% group $(n=7)$ was fed a HFC diet supplemented with $2.5 \%(w / w)$ EMP, and the EMP5\% group $(n=7)$ was fed a HFC diet supplemented with $5 \%(w / w)$ EMP. The HFC diet was prepared by mixing MF with $28.75 \%(w / w)$ palm oil, $1.25 \%(w / w)$ cholesterol, and $0.5 \%(w / w)$ sodium cholate [11]. EMP was provided by L.S Corporation, Tokyo, Japan [12]. Mackerel meat was powdered and heated at $90{ }^{\circ} \mathrm{C}$, and then was digested with proteases from Bacillus subtilis and Aspergillus oryzae. The hydrolysate was further digested with pancreatin and leucine aminopeptidase. After the subsequent processes of filtration, separation of oils and fats, concentration, removal of metals, and finally desiccation, approximately $20 \mathrm{~g}$ of EMP powder was obtained from $400 \mathrm{~g}$ of mackerel meat. Ultimately, $100 \mathrm{~g}$ of the EMP powder contained $90 \mathrm{~g}$ of protein, $0.2 \mathrm{~g}$ of lipid, $9.1 \mathrm{~g}$ of ash, and $951 \mu \mathrm{g}$ of selenium. The proximate dietary compositions of each diet fed to the rats are shown in Table 1. Daily energy intake and body weight were monitored throughout the study.

At 18 weeks of age, the rats were fasted for 6 hours and sacrificed under anaesthesia with pentobarbital sodium. Blood samples were taken from the inferior vena cava or heart and kept at $20^{\circ} \mathrm{C}$ until analysis. The epididymal fat pad and liver were removed, washed in cold saline, and weighed. Liver tissues were either placed in $10 \%$ neutral buffered formalin or snap frozen in liquid nitrogen and stored at $-80^{\circ} \mathrm{C}$.

Table 1 Proximate dietary compositions.

\begin{tabular}{lccccc}
\hline Ingredient\Group & Control & HFC & EMP1\% & EMP2.5\% & EMP5\% \\
\hline Water (g) & 7.90 & 5.49 & 5.46 & 5.42 & 5.35 \\
Crude protein (g) & 23.10 & 16.05 & 16.79 & 17.89 & 19.73 \\
Crude lipid (g) & 5.10 & 3.54 & 3.51 & 3.46 & 3.37 \\
Crude ash (g) & 5.80 & 4.03 & 4.08 & 4.16 & 4.28
\end{tabular}




\begin{tabular}{lccccc} 
Crude fiber (g) & 2.80 & 1.95 & 1.93 & 1.90 & 1.85 \\
Nitrogen-free extract (g) & 55.30 & 38.43 & 38.04 & 37.45 & 36.48 \\
Palm oil (g) & 0.00 & 28.75 & 28.46 & 28.02 & 27.29 \\
Cholesterol (g) & 0.00 & 1.25 & 1.24 & 1.22 & 1.19 \\
Sodium cholate (g) & 0.00 & 0.50 & 0.49 & 0.49 & 0.47 \\
\hline Total (g) & 100.00 & 100.00 & 100.00 & 100.00 & 100.00 \\
\hline Protein energy ratio (\%) & 25.70 & 12.63 & 13.25 & 14.18 & 15.76 \\
Lipid energy ratio (\%) & 12.77 & 57.15 & 56.74 & 56.13 & 55.10 \\
Carbohydrate energy ratio (\%) & 61.53 & 30.23 & 30.01 & 29.69 & 29.14 \\
\hline Energy (kcal/100g) & 359.50 & 508.60 & 507.03 & 504.68 & 500.76 \\
\hline
\end{tabular}

Control group rats were fed MF diet as a normal diet.

HFC, high-fat and high-cholesterol diet; EMP, peptides obtained by enzymatic decomposition of mackerel; EMP1\%, EMP2.5\% and EMP5\%, HFC diet supplemented with 1\%, 2.5\% and 5\% $(w / w)$ EMP, respectively.

\subsection{Serum biochemical analysis}

Serum triglyceride (TG), free fatty acid (FFA), total cholesterol (TC), free cholesterol (FC), glucose, aspartate aminotransferase (AST), and alanine aminotransferase (ALT) levels were determined using Triglyceride E test Wako, NEFA C test Wako, Cholesterol E test Wako, Free cholesterol E test Wako, Glucose C II test Wako, and Transaminase C II test Wako (Wako Pure Chemical Industries, Osaka, Japan), respectively. Serum insulin, leptin, and adiponectin levels were measured using a rat insulin enzyme-linked immunosorbent assay (ELISA) kit (Morinaga Institute of Biological Science Inc., Yokohama, Japan), a mouse/rat leptin ELISA kit (Morinaga Institute of Biological Science Inc.), and a mouse/rat adiponectin ELISA kit (Otsuka Pharmaceuticals Co., Ltd., Tokyo, Japan), respectively.

\subsection{Hepatic lipid analysis}

Hepatic lipids were extracted from the frozen liver using the method of Folch et al. [13]. The extract was dissolved in isopropanol and analyzed for TG and TC with a kit, as described above.

\subsection{Histopathological examinations}

Liver tissues stored in 10\% neutral-buffered formalin were embedded in paraffin, sectioned at 4 $\mu \mathrm{m}$, and processed for hematoxylin-eosin (HE) and Azan staining for histopathological examination. Histological steatosis (0-3), lobular inflammation (0-3), and hepatocyte ballooning (0-2) were assessed semi-quantitatively to determine the NAFLD activity score (NAS) according to the NASH Clinical Research Network Scoring System [14]. NAS scores $\geq 5$ and $\leq 2$ were considered diagnostic and not diagnostic, respectively, for steatohepatitis. Liver fibrosis (0-4) was also assessed according to this system [13]. For the immunohistochemical analysis of 4-hydroxynonenal (4-HNE), deparaffinized liver specimens were incubated with rabbit polyclonal antibodies against 4-HNE (OXIS International, Foster City, CA, USA) to detect oxidative stress-induced lipid peroxidation. Normal rabbit serum was used as a negative control. EnVision peroxidase was used as the second antibody (DAKO, Glostrup, Denmark), and 3,3' diaminobenzidine (DAB) was used as the substrate. 
The intensity of 4-HNE was scored as 0,1 , and 2, indicating no positive staining or slightly positive in the perivenular zone, slightly positive in the periportal zone, and positive staining in hepatocytes or strongly positive in the periportal zone, respectively. All histopathological examinations were performed by a pathologist (K.T.) who was blinded to the experimental and serological data.

\subsection{Quantification of MRNA using real-time polymerase chain reaction}

Total RNA from the liver was extracted using RNAiso Plus (Takara Bio, Otsu, Japan) according to the manufacturer's instructions. RNA was reverse-transcribed to cDNA templates using a commercial kit (PrimeScript RT Master Mix, Takara Bio). Real-time polymerase chain reaction (PCR) analysis was performed as described previously [11]. Specific primers were designed using the Primer-BLAST primer designing tool (National Center for Biotechnology Information [NCBI], Bethesda, MD, USA) and were synthesized by Greiner Bio-One Japan (Tokyo, Japan) (Table 2).

Table 2 Primer sequences for real-time polymerase chain reaction.

\begin{tabular}{|c|c|c|}
\hline Primer & Forward (5' to $\left.3^{\prime}\right)$ & Reverse (5' to $\left.3^{\prime}\right)$ \\
\hline COL1A1 & GCGTAGCCTACATGGACCAA & AAGTTCCGGTGTGACTCGTG \\
\hline$\underline{T G F-B}$ & CTTTGTACAACAGCACCCGC & TAGATTGCGTTGTTGCGGTC \\
\hline$\underline{\alpha-S M A}$ & GCCAAGAAGACATCCCTGAAGT & TGTGGCAGATACAGATCAAGCAT \\
\hline$\underline{T N F-\alpha}$ & TGATCGGTCCCAACAAGGA & TGGGCTACGGGCTTGTCA \\
\hline$M C P-1$ & TCTGTCACGCTTCTGGGCCTGT & GGGGCATTAACTGCATCTGGCTGAG \\
\hline$\underline{I L-16}$ & CTCCAGTCAGGCTTCCTTGTG & GGTCATTCTCCTCACTGTCGAAA \\
\hline$\underline{I L-6}$ & GATACCACCCACAACAGACCAGTA & TGCACAACTCTTTTCTCATTTCCA \\
\hline$N F-K B$ & TGACATCATCAACATGAGAAACGA & CCCCAACCCTCAGCAAGTC \\
\hline CYP2E1 & CCCATCCTTGGGAACATTTTT & GCCAAGGTGCAGTGTGAACA \\
\hline $\mathrm{HO}-1$ & CACAGGGTGACAGAAGAGGCTAA & GGGACTCTGGTCTTTGTGTTCCT \\
\hline$G P X-1$ & GCTGCTCATTGAGAATGTCG & GAATCTCTTCATTCTTGCCATT \\
\hline MnSOD & GACCTGCCTTACGACTATG & TACTTCTCCTCGGTGACG \\
\hline$\underline{L X R-\alpha}$ & CAGGACCAGCTCCAAGTAGA & GAACATCAGTCGGTCGTGG \\
\hline MTP & CAAGCTCAAGGCAGTGGTTG & AGCAGGTACATCGTGGTGTC \\
\hline FAS & CAACATTGACGCCAGTTCCG & TTCGAGCCAGTGTCTTCCAC \\
\hline GPAT & GCTACCTGAAGGTGAGCCAG & AGGTACTCAGACTCCGGGAC \\
\hline$\underline{C P T-1}$ & AACCTCGGACCCAAATTGC & GGCCCCGCAGGTAGATATATT \\
\hline AOX & CCACTGAACAAAACAGAGGTCC & GTCCCAGGGAAACTTCAAAGC \\
\hline
\end{tabular}

All quantifications were normalized using the housekeeping gene glyceraldehyde 3-phosphate dehydrogenase (GAPDH). For studies in rats, hepatic expressions of genes involved in fibrosis [collagen type I alpha 1 (COL1A1), transforming growth factor- $\beta$ (TGF- $\beta$ ), and $\alpha$-smooth muscle actin $(\alpha-S M A)$ ], inflammation [tumor necrosis factor- $\alpha$ (TNF- $\alpha)$, monocyte chemoattractant

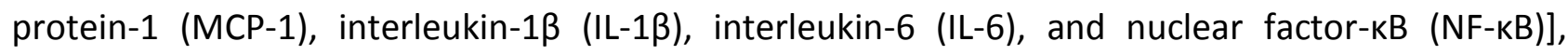
oxidative stress [cytochrome P450 family 2 subfamily E polypeptide 1 (CYP2E1), heme oxygenase-1 (HO-1), glutathione peroxidase-1 (GPX-1), and manganese superoxide dismutase (MnSOD)], 
cholesterol metabolism [liver X receptor- $\alpha(\mathrm{LXR}-\alpha)$ ], and lipid metabolism [microsomal triglyceride transfer protein (MTP), fatty acid synthase (FAS), glycerol-3-phosphate acyltransferase (GPAT), carnitine palmitoyltransferase-1 (CPT-1), and acyl-CoA oxidase (AOX)] were quantified. All data were expressed as a fold change relative to the control group.

\subsection{Statistical analysis}

All values were expressed as mean \pm standard error $(\mathrm{SE})$. Differences between groups were tested for statistical significance using one-way analysis of variance (ANOVA), followed by Scheffe's post hoc test, chi-square test, or Fisher's exact probability test. Correlations between two variables were determined by Spearman's rank correlation coefficient. All analyses were performed using IBM SPSS statistics software program, version 24 (IBM Co., Somers, NY, USA) on a Windows computer. A p value of less than 0.05 was considered to be statistically significant.

\section{Results}

\subsection{Cumulative energy intake, body weight, and relative organ weights}

During the 9-week study (9 to 18 weeks of age), cumulative energy intake was significantly higher in the EMP1\% group than in the control and EMP5\% groups ( $p=0.003$ and 0.023 , respectively), whereas body weight at 18 weeks of age and weight gain during the 9-week study were not significantly different among the groups. The liver weight/body weight ratio at 18 weeks of age was significantly higher in the HFC, EMP1\%, EMP2.5\%, and EMP5\% groups than in the control group $(p<0.001)$, and this ratio was notably similar between the HFC, EMP1\%, EMP2.5\%, and EMP5\% groups. The epididymal fat pad weight/body weight ratio was not significantly different among the groups (Table 3 ).

Table 3 Cumulative energy intake, body weight, relative organ weights, serum parameters and hepatic lipid concentrations at 18 weeks of age.

\begin{tabular}{lccccc}
\hline Item\Group & $\begin{array}{c}\text { Control } \\
(\mathrm{n}=6)\end{array}$ & $\begin{array}{c}\text { HFC } \\
(\mathrm{n}=6)\end{array}$ & $\begin{array}{c}\text { EMP1\% } \\
(\mathrm{n}=6)\end{array}$ & $\begin{array}{c}\text { EMP2.5\% } \\
(\mathrm{n}=7)\end{array}$ & $\begin{array}{c}\text { EMP5\% } \\
(\mathrm{n}=7)\end{array}$ \\
\hline Cumulative energy intake (kcal) & $5187 \pm 86^{\mathrm{a}}$ & $5907 \pm 165^{\mathrm{ab}}$ & $6253 \pm 277^{\mathrm{b}}$ & $5782 \pm 130^{\mathrm{ab}}$ & $5426 \pm 97^{\mathrm{a}}$ \\
Final body weight (g) & $511 \pm 11$ & $525 \pm 10$ & $527 \pm 13$ & $506 \pm 13$ & $489 \pm 9$ \\
Body weight gain (g) & $210 \pm 9$ & $223 \pm 10$ & $225 \pm 12$ & $208 \pm 12$ & $191 \pm 7$ \\
Liver/body weight (\%) & $2.8 \pm 0.1^{\mathrm{a}}$ & $5.5 \pm 0.2^{\mathrm{b}}$ & $5.5 \pm 0.1^{\mathrm{b}}$ & $5.4 \pm 0.2^{\mathrm{b}}$ & $5.6 \pm 0.2^{\mathrm{b}}$ \\
Epididymal fat pad/body weight (\%) & $1.9 \pm 0.1$ & $2.2 \pm 0.2$ & $2.2 \pm 0.1$ & $2.3 \pm 0.1$ & $1.7 \pm 0.2$ \\
Serum triglyceride (mg/dL) & $72 \pm 8$ & $55 \pm 8$ & $52 \pm 3$ & $63 \pm 5$ & $52 \pm 9$ \\
Serum free fatty acid (mEq/L) & $0.28 \pm 0.06$ & $0.28 \pm 0.08$ & $0.28 \pm 0.04$ & $0.26 \pm 0.04$ & $0.22 \pm 0.04$ \\
Serum total cholesterol (mg/dL) & $65 \pm 5^{\mathrm{a}}$ & $91 \pm 4^{\mathrm{ab}}$ & $83 \pm 5^{\mathrm{ab}}$ & $92 \pm 9^{\mathrm{ab}}$ & $95 \pm 6^{\mathrm{b}}$ \\
Serum free cholesterol (mg/dL) & $22 \pm 2^{\mathrm{a}}$ & $27 \pm 2^{\mathrm{a}}$ & $26 \pm 2^{\mathrm{a}}$ & $29 \pm 2^{\mathrm{a}}$ & $40 \pm 3^{\mathrm{b}}$ \\
Serum glucose (mg/dL) & $134 \pm 8$ & $152 \pm 14$ & $130 \pm 9$ & $159 \pm 15$ & $181 \pm 45$ \\
Serum insulin (ng/mL) & $6.4 \pm 1.3$ & $7.2 \pm 1.1$ & $4.6 \pm 1.5$ & $5.7 \pm 0.9$ & $6.1 \pm 1.0$ \\
Serum AST (IU/L) & $56 \pm 12$ & $137 \pm 22$ & $111 \pm 19$ & $114 \pm 13$ & $120 \pm 22$ \\
Serum ALT (IU/L) & $11 \pm 1^{\mathrm{a}}$ & $44 \pm 8^{\mathrm{ab}}$ & $51 \pm 13^{\mathrm{ab}}$ & $83 \pm 8^{\mathrm{b}}$ & $92 \pm 14^{\mathrm{b}}$ \\
\hline
\end{tabular}




\begin{tabular}{lccccc} 
Serum leptin $(\mathrm{ng} / \mathrm{mL})$ & $7.4 \pm 0.5^{\mathrm{ab}}$ & $9.4 \pm 1.1^{\mathrm{b}}$ & $5.3 \pm 0.6^{\mathrm{ac}}$ & $5.5 \pm 0.4^{\mathrm{ac}}$ & $3.6 \pm 0.4^{\mathrm{c}}$ \\
Serum adiponectin $(\mu \mathrm{g} / \mathrm{mL})$ & $4.4 \pm 0.4$ & $4.0 \pm 0.6$ & $3.0 \pm 0.4$ & $3.5 \pm 0.4$ & $4.0 \pm 0.5$ \\
Hepatic triglyceride $(\mathrm{mg} / \mathrm{g}$ tissue) & $22 \pm 4^{\mathrm{a}}$ & $95 \pm 14^{\mathrm{b}}$ & $127 \pm 18^{\mathrm{bc}}$ & $151 \pm 4^{\mathrm{c}}$ & $150 \pm 10^{\mathrm{c}}$ \\
Hepatic total cholesterol $(\mathrm{mg} / \mathrm{g}$ tissue) & $3.8 \pm 0.2^{\mathrm{a}}$ & $78.8 \pm 9.9^{\mathrm{b}}$ & $101.0 \pm 12.7^{\mathrm{b}}$ & $75.6 \pm 4.9^{\mathrm{b}}$ & $78.6 \pm 12.2^{\mathrm{b}}$ \\
\hline
\end{tabular}

Values are expressed as means \pm SE.

${ }^{a b c}$ Values not sharing the same lowercase letter in a row are significantly different among groups ( $p<0.05$ ). HFC, high-fat and high-cholesterol diet; EMP, peptides obtained by enzymatic decomposition of mackerel; EMP1\%, EMP2.5\% and EMP5\%, HFC diet supplemented with 1\%, $2.5 \%$ and $5 \%(\mathrm{w} / \mathrm{w}) \mathrm{EMP}$, respectively; AST, aspartate aminotransferase; ALT, alanine aminotransferase.

\subsection{Serum and hepatic biochemical parameters}

At 18 weeks of age, serum TG, FFA, glucose, insulin, AST, and adiponectin levels were not significantly different among the groups. Serum TC levels were significantly higher in the EMP5\% group than in the control group $(p=0.039)$, and serum FC levels were significantly higher in the EMP5\% group than in the control, HFC, EMP1\%, and EMP2.5\% groups $(p<0.001,0.005,0.004$, and 0.018 , respectively). Serum ALT levels were significantly lower in the control group than in the EMP2.5\% and EMP5\% groups ( $p=0.001$ and $<0.001$, respectively). Serum leptin levels were significantly higher in the HFC group than in the EMP1\%, EMP2.5\%, and EMP5\% groups ( $p=0.006$, 0.005 , and $<0.005$, respectively); the serum leptin levels were also significantly higher in the control group than in the EMP5\% group $(p=0.007)$. Hepatic TG values were significantly lower in the control group than in the HFC, EMP1\%, EMP2.5\%, and EMP5\% groups ( $p=0.003,<0.001$, $<0.001$, and $<0.001$, respectively), and were also significantly lower in the HFC group than in the EMP2.5\% and EMP5\% groups ( $p=0.025$ and 0.029 , respectively). Hepatic TC values were significantly lower in the control group than in the other 4 groups $(p<0.001)$ (Table 3$)$.

\subsection{Histopathological findings of the liver}

Representative histopathology of the rat liver and histological assessments are shown in Figure 1 and Table 4. No obvious findings of hepatic steatosis, lobular inflammation, hepatocyte ballooning, or fibrosis were seen in any of the rats in the control group. In contrast, severe steatosis (score 3), moderate or severe lobular inflammation (score 2 or 3), and mild or perisinusoidal and portal/periportal fibrosis (score 1a or 2) were observed in all 6 rats of the HFC group, despite no evidence of hepatocyte ballooning. In the EMP1\%, EMP2.5\%, and EMP5\% groups, severe steatosis (score 3) was observed in 19 (95\%) of the rats; moderate or severe lobular inflammation (score 2 or 3 ) was observed in 17 (85\%) of the rats; and perisinusoidal, portal/periportal, or bridging fibrosis (score 2 or 3 ) was observed in 12 (60\%) of the 20 rats. According to the NAS [14], none of 6 rats of the control group (0\%), all of 6 rats of the HFC group (100\%), 5 of 6 rats of the EMP1\% group (83\%), 5 of 7 rats of the EMP2.5\% group (71\%), and 6 of 7 rats of the EMP5\% group (86\%) were diagnosed with NASH (i.e., having a NAS of 5 of greater).

Representative immunohistochemistry of the rat liver and immunohistochemical assessments are also shown in Figure 1 and Table 4. Negative staining (score 0) was seen in all 6 rats of the control group, whereas positive staining (score 2) was observed in 5 of 6 rats (83\%) in the HFC group. In the 20 rats of EMP1\%, EMP2.5\% or EMP5\% group, negative staining (score 0 ) was 
observed in 5 (25\%) rats, and positive staining (score 2) was also observed in 5 (25\%) rats. The 4HNE staining score was significantly correlated with the histological lobular inflammation score and the diagnostic result of NAS ( $p=0.005$ and $p=0.001$, respectively) (Table 5 ).

Control
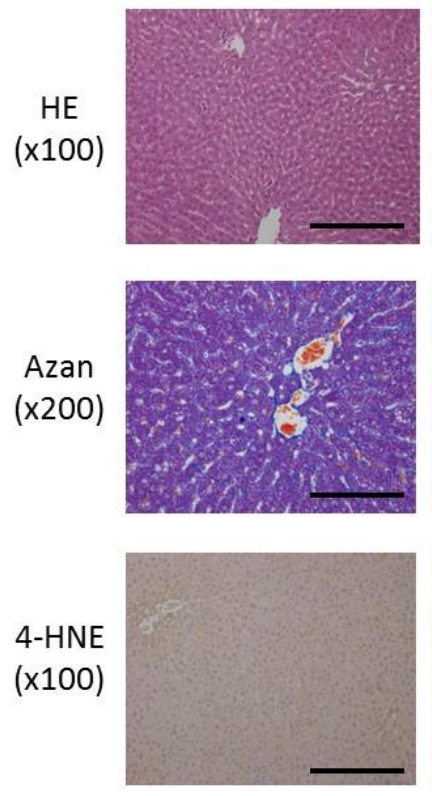

HFC
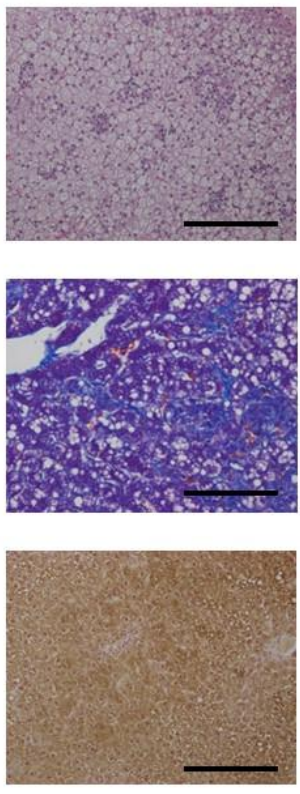

EMP1\%
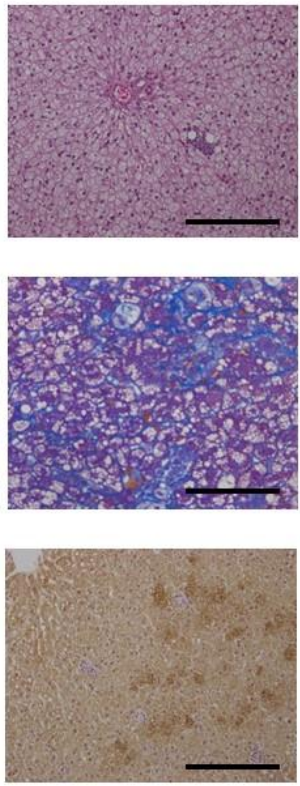

EMP2.5\%
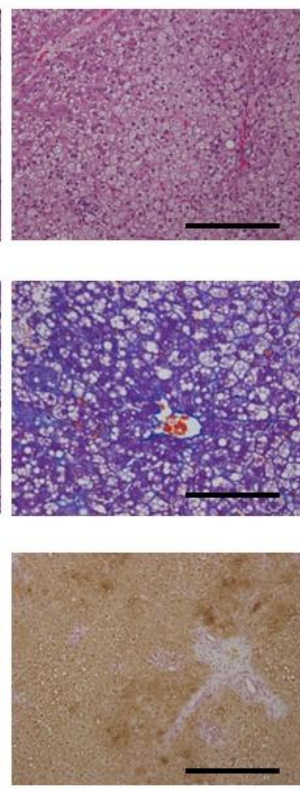

EMP5\%
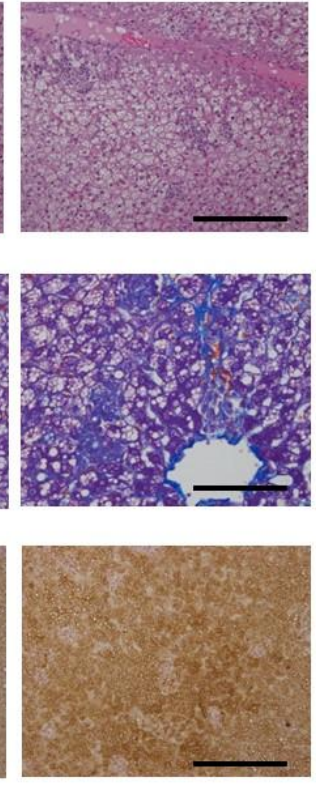

Figure 1 Representative histopathology of the rat liver in the control, HFC, EMP1\%, EMP2.5\%, and EMP5\% groups at 18 weeks of age. Upper panels: HE-stained sections (original magnification, $\mathrm{x} 100$; scale bars $=400 \mu \mathrm{m}$ ). Middle panels: Azan-stained sections (original magnification, $\times 200$; scale bars $=200 \mu \mathrm{m}$ ). Lower panels: Immunohistochemical 4-HNE-stained sections (original magnification, $x 100$; scale bars $=400 \mu \mathrm{m}$ ). HFC indicates high-fat and high-cholesterol diet; EMP indicates peptides obtained by enzymatic decomposition of mackerel; EMP1\%, EMP2.5\%, and EMP5\% were fed HFC diet supplemented with $1 \%, 2.5 \%$, and $5 \%(\mathrm{w} / \mathrm{w})$ EMP, respectively.

Table 4 Histopathological and immunohistochemical assessments of the liver at 18 weeks of age.

\begin{tabular}{lcccccc}
\hline Item\Group & Score & $\begin{array}{c}\text { Control } \\
(\mathbf{n}=6)\end{array}$ & $\begin{array}{c}\text { HFC } \\
(\mathbf{n}=6)\end{array}$ & $\begin{array}{c}\text { EMP1\% } \\
(\mathbf{n}=6)\end{array}$ & $\begin{array}{c}\text { EMP2.5\% } \\
(\mathbf{n}=\mathbf{7})\end{array}$ & $\begin{array}{c}\text { EMP5\% } \\
(\mathbf{n}=\mathbf{7})\end{array}$ \\
\hline Steatosis* & 0 & 6 & 0 & 0 & 0 & 0 \\
& 2 & 0 & 0 & 0 & 0 & 1 \\
Lobular inflammation* & 3 & 0 & 6 & 6 & 7 & 6 \\
& 0 & 6 & 0 & 0 & 0 & 0 \\
& 1 & 0 & 0 & 1 & 2 & 0 \\
Hepatocyte ballooning* & 2 & 0 & 5 & 2 & 3 & 5 \\
NAFLD activity score (NAS)* & 0 & 0 & 1 & 3 & 2 & 2 \\
& 3 & 6 & 6 & 6 & 7 & 7 \\
& $3-4$ & 0 & 0 & 1 & 0 & 0
\end{tabular}




\begin{tabular}{lcccccc} 
& $5-8$ & 0 & 6 & 5 & 5 & 6 \\
Fibrosis* & $0-1$ & 6 & 5 & 2 & 5 & 1 \\
\multirow{3}{*}{ 4-HNE staining } & $2-3$ & 0 & 1 & 4 & 2 & 6 \\
& 0 & 6 & 0 & 1 & 2 & 2 \\
& 1 & 0 & 1 & 3 & 5 & 2 \\
& 2 & 0 & 5 & 2 & 0 & 3 \\
\hline
\end{tabular}

Values are expressed as the number of rats.

*Scores are according to the NASH Clinical Research Network Scoring System proposed by Kleiner et al. [14].

HFC, high-fat and high-cholesterol diet; EMP, peptides obtained by enzymatic decomposition of mackerel; EMP1\%, EMP2.5\% and EMP5\%, HFC diet supplemented with 1\%, 2.5\% and 5\% (w/w) EMP, respectively; 4-HNE, 4-hydroxynonenal.

Table 5 Correlations between 4-HNE staining score and histological lobular inflammation score or NAS score.

\begin{tabular}{llll}
\hline Item \4-HNE staining score & $\mathbf{0}$ & $\mathbf{1}$ & $\mathbf{2}$ \\
\hline Histological lobular inflammation* & & & 0 \\
Score 0 & 6 & 0 & 0 \\
Score 1 & 1 & 2 & 8 \\
Score 2 & 2 & 5 & 2 \\
Score 3 & 2 & 4 & \\
NAFLD activity score (NAS)* & & & 0 \\
0-2 (non-NASH) & 6 & 0 & 0 \\
3-4 (borderline) & 1 & 3 & 10 \\
5-8 (NASH) & 4 & 8 & \\
\hline
\end{tabular}

Values are expressed as the number of rats.

*Scores are according to the NASH Clinical Research Network Scoring System proposed by Kleiner et al. [14].

4-HNE, 4-hydroxynonenal; NASH, nonalcoholic steatohepatitis.

\subsection{Hepatic mRNA expression}

To reveal the effect of EMP on NASH on a molecular level, we evaluated key molecular markers of fibrosis, inflammation, oxidative stress, and cholesterol and lipid metabolism in the liver. As shown in Figure 2, the mRNA levels of COL1A1 and $\alpha-S M A$, which are both involved in fibrogenesis, tended to be lower in the EMP1\% and EMP2.5\% groups than in the HFC group; however, the mRNA level of TGF-B (which is a key inducer of fibrogenesis), was similar between these groups and the HFC group. The mRNA levels of $T N F-\alpha$ and $I L-16$, which are involved in inflammation, tended to be higher in the EMP groups (EMP1\%, EMP2.5\%, and EMP5\%) than the HFC group in an EMP-dose dependent manner. The mRNA levels of MCP-1 and $I L-6$, which are also involved in inflammation, tended to be lower in the EMP1\% group and EMP2.5\% group compared to the HFC group, but were the highest in the EMP5\% group. In contrast, the mRNA level of NF- $K B$, which is a central regulator of inflammation, tended to be lower in the EMP groups than in the HFC group in 
an EMP-dose dependent manner. The mRNA levels of CYP2E1 and MnSOD, which is a member of the cytochrome P450 mixed-function oxidase system and a member of the iron/manganese superoxide dismutase family, respectively, did not significantly differ between the HFC group and

A Fibrosis

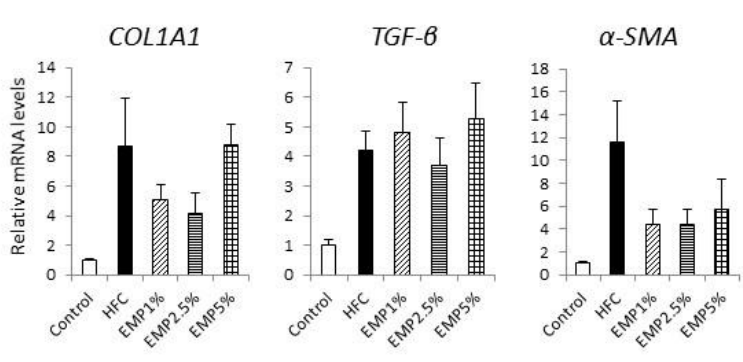

B Inflammation

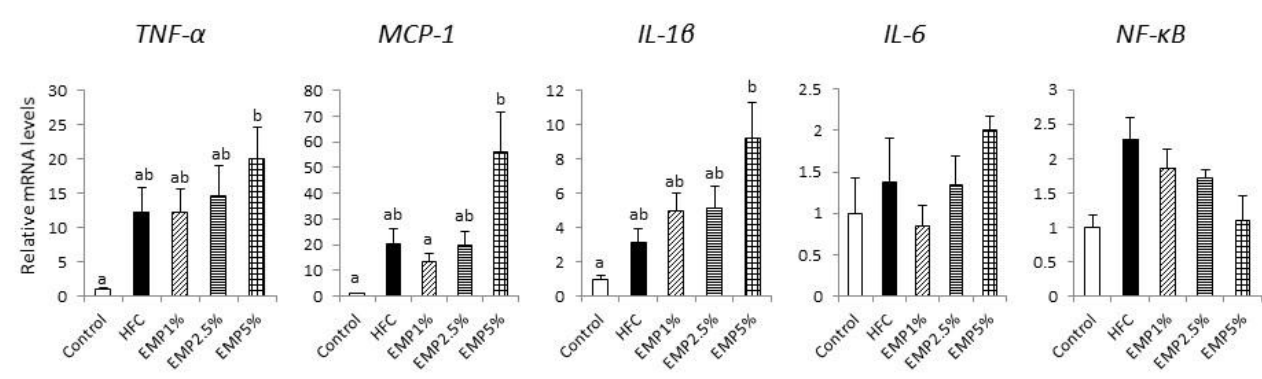

C Oxidative stress

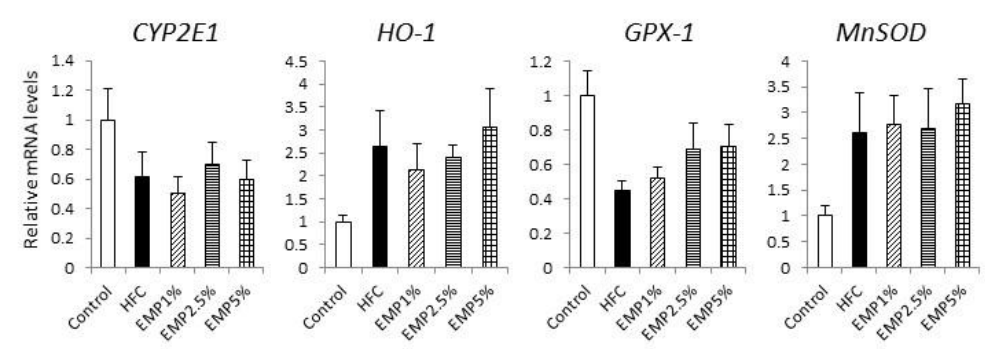

D Cholesterol or lipid metabolism
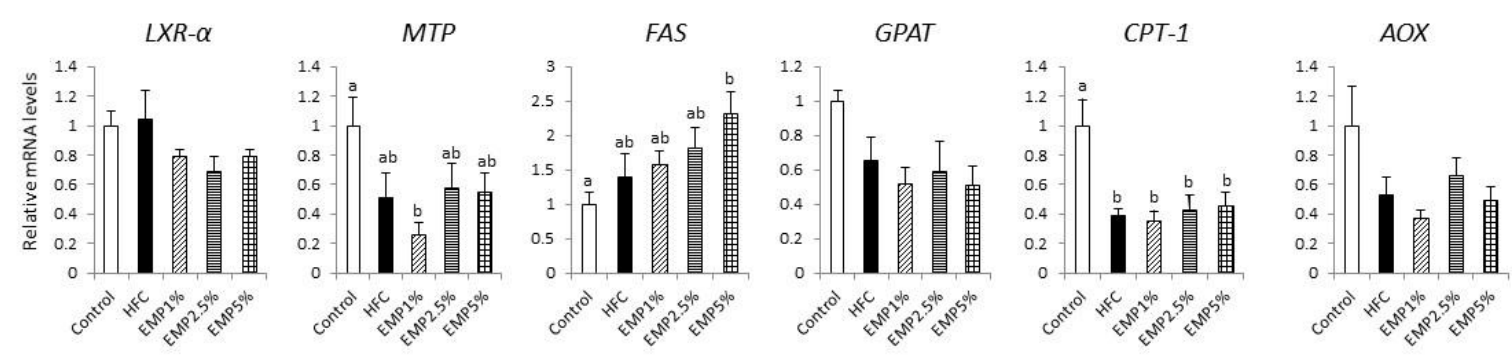

Figure 2 Hepatic gene expression involved in (A) fibrosis, (B) inflammation, (C) oxidative stress, and (D) cholesterol or lipid metabolism in the control, HFC, EMP1\%, 
EMP2.5\%, and EMP5\% groups ( $n=6-7 /$ group) at 18 weeks of age. mRNA levels are expressed relative to the control group (mean $\pm \mathrm{SE}$ ). abValues not sharing the same lowercase letter in a row are significantly different among groups $(p<0.05)$. HFC indicates high-fat and high-cholesterol diet; EMP indicates peptides obtained by enzymatic decomposition of mackerel; EMP1\%, EMP2.5\%, and EMP5\% were fed HFC diet supplemented with $1 \%, 2.5 \%$, and $5 \%(w / w)$ EMP, respectively.

EMP groups. The mRNA level of $\mathrm{HO}-1$, which is an inducible enzyme in response to oxidative stress, tended to be lower in the EMP1\% group than in the HFC group. In contrast to this, the mRNA level of GPX-1, which is an intracellular antioxidant enzyme, tended to be higher in the EMP groups than in the HFC group in an EMP-dose dependent manner, although the difference was not statistically significant. The mRNA level of $L X R-\alpha$, which is involved in hepatic lipid homeostasis, tended to be lower in the EMP groups than in the HFC group. The mRNA level of MTP, which is a rate-limiting protein in the synthesis and excretion of very-low-density lipoprotein (VLDL) from the liver, was lower in the EMP1\% group than in the control group. The mRNA level of FAS, which is a multi-enzyme protein that catalyzes fatty acid synthesis, tended to be higher in the EMP groups than in the HFC group in an EMP-dose dependent manner. The mRNA levels of GPAT, CPT-1, and $A O X$, which are involved in lipid metabolism, were not significantly different between the HFC group and the EMP groups.

The mRNA level of TGF- $B$ was positively correlated with histological hepatic fibrosis $(p=0.001, r$ $=0.553$ ). The mRNA levels of NF-KB and HO-1 were positively correlated with histological lobular inflammation of the liver ( $p=0.003, r=0.513$ and $p=0.005, r=0.481$, respectively). The mRNA level of GPX-1 was negatively correlated with immunohistochemical 4-HNE staining (Figure 3 ).
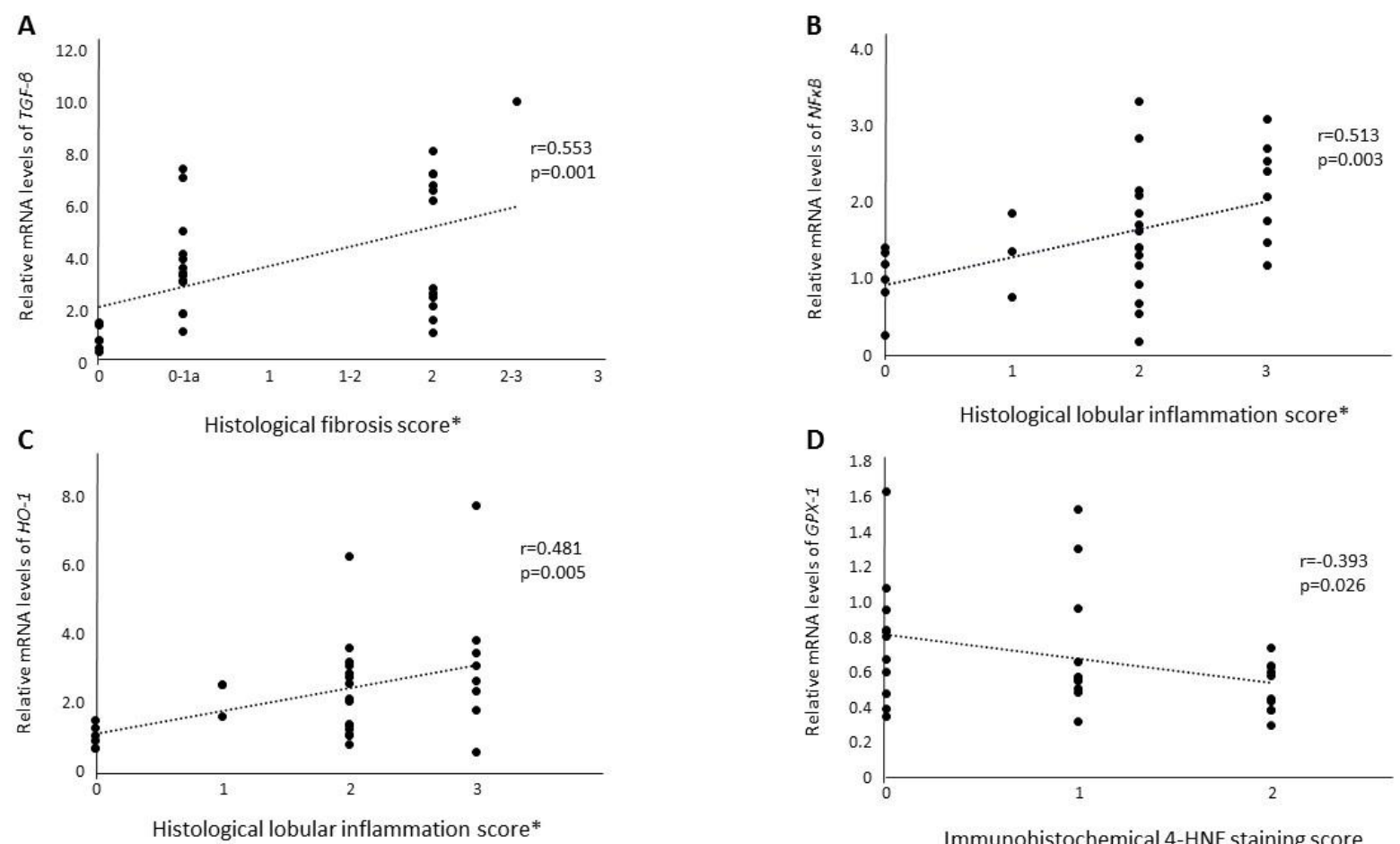

Immunohistochemical 4-HNE staining score 
Figure 3 Correlations between hepatic histological findings and gene expressions. Histological fibrosis score is positively correlated with the relative mRNA level of TGF- 6 (A). Histological lobular inflammation scores are positively correlated with the relative mRNA levels of $N F K B(B)$ and $H O-1$ (C), respectively. Immunohistochemical 4-HNE staining score is negatively correlated with the relative mRNA level of GPX-1 (D). *Scores are according to the NASH Clinical Research Network Scoring System proposed by Kleiner et al. [14].

\section{Discussion}

The daily doses of EMP in this study were determined based on human doses. The average daily selenium intake of Japanese people is reported to be approximately $100 \mu \mathrm{g}$ [15]. Because EMP contains $951 \mathrm{\mu g}$ selenium in $100 \mathrm{~g}$, daily doses of EMP1\%, 2.5\%, and 5\% in rats would be equivalent to daily doses of approximately 77,193 , and $385 \mu \mathrm{g}$ in humans, respectively, after taking into account the human equivalent dose.

In this study, there were no significant differences in liver/body weight, epididymal fat pad weight/body weight, or serum metabolic parameters such as serum glucose, insulin, or adiponectin at 18 weeks of age between the HFC group and the EMP groups; however, serum leptin levels were lower in EMP groups than in the HFC group. Leptin possesses a number of effects on the immune system and plays an important role in modulating inflammatory responses, regulation of food intake, and energy expenditure [16]. Indeed, serum leptin levels were negatively correlated with serum ALT levels in this study ( $p=0.003, r=-0.510$, data not shown). No significant difference in epididymal fat pad weight/body weight, serum triglyceride levels, or free cholesterol levels between the HFC groups and the EMP groups was detected; this indicates that the leptin-lowering component of EMP may not largely affect obesity or visceral fat tissue. Further studies examining the insulin-leptin axis and the role of endotoxins are needed to determine the mechanism of this effect $[16,17]$.

The serum FC levels were significantly higher in the EMP5\% group than in the control, HFC, EMP1\%, and EMP2.5\% groups in this study. The reason for this result is unclear. Lecithin cholesterol acyltransferase (LCAT) is synthesized by the liver, and esterifies FC on the surface of $\mathrm{HDL}$, forming cholesteryl esters [18]. Because the histological hepatic fibrosis was more progressive in the EMP5\% group, it is conceivable that LCAT synthesis and esterification of FC can be stagnated.

Histopathological findings of the liver revealed that all 6 rats in the HFC group were diagnosed with NASH; contrastingly, 4 out of 20 rats $(20 \%)$ in the EMP groups had borderline scores according to the NASH Clinical Research Network Scoring System [14] and were not diagnosed as fully-developed NASH in this study. Because the histopathological findings of steatosis and hepatocyte ballooning were similar between the HFC group and the EMP groups, this difference in the NASH diagnostic rate between the HFC group and the EMP groups ( $100 \%$ vs $80 \%$ ) was mainly due to the histopathological lobular inflammation score. Moreover, immunohistochemical staining intensities of 4-HNE tended to be lower in the EMP groups than the HFC group; the 4-HNE staining score was significantly correlated with the histological lobular inflammation score and the diagnostic result of NAS. Oxidative stress seems to be one of the most important mechanisms leading to hepatic injury in NAFLD, as augmented generation of reactive oxygen species (ROS) can 
induce lipid peroxidation, leading to inflammation [19]. Thus, our results indicate that EMP reduced oxidative stress, which decreased lobular inflammation and NASH progression.

The TGF- $\beta$ pathway is a significant modulator of $\operatorname{COL} 1 A 1$, the type I collagen gene $[20,21]$, while Smads are a family of proteins that operate downstream of various members of the TGF- $\beta$ superfamily [20]. In this study, the mRNA level of TGF- $B$ was positively correlated with histological hepatic fibrosis. mRNA levels of TGF- $B$ in the EMP groups were similar to that in the HFC group, and mRNA levels of COL1A1 and $\alpha$-SMA tended to be lower in the EMP1\% and EMP2.5\% groups than in the HFC group. However, histological hepatic fibrosis was more progressive in the EMP groups than in the HFC group. The reason for these discrepancies is not clear but may be attributable to the non-Smad signaling pathways [22].

Antioxidants can inhibit NF-KB activation [23]. In this study, the mRNA levels of NF- $K B$ were positively correlated with histological lobular inflammation of the liver and tended to be lower in the EMP groups (EMP1\%, EMP2.5\%, and EMP5\%) than in the HFC group in an EMP-dose dependent manner. In contrast, the mRNA level of the intracellular antioxidant enzyme GPX-1 was negatively correlated with immunohistochemical 4-HNE staining and tended to be higher in the EMP groups than in the HFC group in an EMP-dose dependent manner, although the difference was not statistically significant. These results indicate that the lobular inflammation in EMP groups can be reduced by NF-KB suppression via GPX-1 expression. However, the histological lobular inflammation score tended to be lower in the EMP1\% and EMP2.5\% groups but not in the EMP5\% group. The reason for this discrepancy is not obvious, but inflammation-related cytokines such as TNF- $\alpha$, MCP1, IL-1 $\beta$, and IL- 6 in the EMP5\% group can be induced by non-NF-KB signaling pathways, which may offer an explanation [24].

Our results from the histological fibrosis score, NAS score, and hepatic mRNA expressions indicate that the EMP5\% group did not reveal a preventive effect on NASH progression. Supranutritional selenium intake or high plasma selenium concentration was reported to be associated with type 2 diabetes or insulin resistance $[8,25,26]$. Selenium supplementation induces selenoproteins such as intracellular antioxidant enzyme GPX-1 and selenium transporter selenoprotein $\mathrm{P}$, which are both implicated in insulin resistance due to the impairment of insulin signaling in the liver and skeletal muscle [8, 25, 27-29]. In this study, the mRNA level of HO-1, which is an inducible enzyme in response to oxidative stress, was positively correlated with histological lobular inflammation of the liver and tended to be lower in the EMP1\% group but higher in the EMP5\% group when compared to the HFC group. ROS can induce lipid peroxidation, leading to fibrogenesis through the activation of stellate cells, and also inhibit hepatocyte secretion of VLDL, which induces liver fat accumulation [19]. Malaguarnera et al. reported that a significant correlation between the increased levels of $\mathrm{HO}-1$ and lipid peroxidation as well as the increased $\mathrm{HO}-1$ expression reflected the severity of $\mathrm{NASH}$ [30]. Therefore, 5\% (w/w) supplementation of EMP to a HFC diet was an excessive dose for SD rats in the prevention of NASH.

\section{Conclusions}

Our results demonstrate that EMP may possess a preventive effect on NASH progression, presumably through its antioxidative stress activity. The EMP1\% and $2.5 \%$ groups, which were fed a HFC diet supplemented with $1 \%$ and $2.5 \%(w / w)$ EMP for 9 weeks, respectively, were more 
effective in prevention than the EMP5\% group, which was fed a HFC diet supplemented with $5 \%$ (w/w) EMP for 9 weeks. The optimal dose of EMP should be further researched in future studies.

\section{Acknowledgments}

The authors thank Mr. Satoshi Matsumoto, L.S Corporation, for providing peptides obtained by enzymatic decomposition of mackerel and for his helpful advice.

\section{Author Contributions}

Katsuhisa Omagari created the study design, adjusted results, and finalized the manuscript.

Ayumi Fukuda carried out the experiments and performed the data analysis.

Machiko Suga carried out the experiments and performed the data analysis.

Ayumi Ogata carried out the experiments and performed the data analysis.

Shinta Nishioka performed the data interpretation and revised the manuscript.

Kazuhito Suruga carried out PCR for mRNA expression and performed the data interpretation.

Mayuko Ichimura carried out the experiments and data collection.

Koichi Tsuneyama performed the histopathological assessment and corrected the manuscript.

\section{Funding}

This work was supported by research funds from the University of Nagasaki and a research grant from L.S Corporation.

\section{Competing Interests}

Katsuhisa Omagari received a research grant from L.S Corporation for this work. The other authors have declared that no competing interests exist.

\section{References}

1. Watanabe S, Hashimoto E, Ikejima K, Uto H, Ono M, Sumida Y, et al. Evidence-based clinical practice guidelines for nonalcoholic fatty liver disease/nonalcoholic steatohepatitis. J Gastroenterol. 2015; 50: 364-377.

2. Day CP, James OFW. Steatohepatitis: a tale of two "hits"? Gastroenterology. 1998; 114: 842845.

3. Tilg $H$, Moschen AR. Evolution of inflammation in nonalcoholic fatty liver disease: the multiple parallel hits hypothesis. Hepatology. 2010; 52: 1836-1846.

4. Yamashita Y, Yamashita M, lida H. Selenium content in seafood in Japan. Nutrients. 2013; 5: 388-395.

5. Yamashita $Y$, Yamashita $M$. Identification of a novel selenium-containing compound, selenoneine, as the predominant chemical form of organic selenium in the blood of Bluefin tuna. J Biol Chem. 2010; 285: 18134-18138.

6. Yamashita Y, Yabu T, Yamashita M. Discovery of the strong antioxidant selenoneine in tuna and selenium redox metabolism. World J Biol Chem. 2010; 1: 144-150. 
7. KroepfI N, Jensen KB, Francesconi KA, Kuehnelt D. Human excretory products of selenium are natural constituents of marine fish muscle. Anal Bioanal Chem. 2015; 407: 7713-7719.

8. Yamashita M, Yamashita $\mathrm{Y}$, Ando T, Wakamiya J, Akiba S. Identification and determination of selenoneine, 2-selenyl- $\mathrm{N} \alpha, \mathrm{N} \alpha, \mathrm{N} \alpha$-trimetyl-I-histidine, as the major organic selenium in blood cells in a fish-eating population on remote Japanese islands. Biol Trace Elem Res. 2013; 156: 36-44.

9. Tamaru S, Matsumoto S, Yamashita M, Yamashita Y, Maeda R. Evaluation of antioxidant potential on novel component, selenoneine, and peptides obtained by enzymatic decomposition of mackerel. Proceedings of the 70th Annual Meeting of Japan Society of Nutrition and Food Science; 2016 May 13-15, 2016; Kobe, Japan. Tokyo: Japan Society of Nutritional and Food Science (in Japanese).

10. Hase-Tamaru S, Kamigaki T, Kanamaru R, Sato K, Matsumoto S. Antioxidant effect in vitro and cytokine secretion in RAW264 cells of mackerel peptides. Proceedings of the 72nd Annual Meeting of Japan Society of Nutritional and Food Science; 2018 May 11-13; Okayama, Japan. Tokyo: Japan Society of Nutritional and Food Science (in Japanese).

11. Ichimura M, Kawase M, Masuzumi M, Sakaki M, Nagata Y, Tanaka K, et al. High-fat and highcholesterol diet rapidly induces non-alcoholic steatohepatitis with advanced fibrosis in Sprague-Dawley rats. Hepatol Res. 2015; 45: 458-469.

12. Sato K, Ejima A. Identification of indigestible peptides in enzyme hydrolysate of mackerel meat with hepatoprotective activity. Proceedings of the International Society for Nefutraceuticls and Functional Foods Annual General Meeting; 2017 October 22-25; Gunsan, the Republic of Korea.

13. Folch J, Lees M, Sloane Stanley GH. A simple method for the isolation and purification of total lipids from animal tissues. J Biol Chem. 1957; 226: 497-509.

14. Kleiner DE, Brunt EM, Van Natta M, Behling C, Contos MJ, Cummings OW, et al. Design and validation of a histological scoring system for nonalcoholic fatty liver disease. Hepatology. 2005; 41: 1313-1321.

15. Yamashita M, Imamura S, Yabu T, Ishihara K, Yamashita Y. Selenium in seafood: Potential nutritional and physiological functions of selenonein. Biomed Res Trace Elements. 2013; 24: 176-184.

16. Landman RE, Puder JJ, Xiao E, Freda PU, Ferin M, Wardlaw SL. Endotoxin stimulates leptin in the human and nonhuman primate. J Clin Endocrinol Metab. 2003; 88: 1285-1291.

17. Gradinaru D, Khaddour H, Margina D, Ungurianu A, Borsa C, Ionescu C, et al. Insulin-leptin axis, cardiometabolic risk and oxidative stress in elderly with metabolic syndrome. Exp Clin Endocrinol Diabetes. 2018; DOI: 10.1055/s-0043-123825.

18. Tosheska Trajkovska K, Topuzovska S. High-density lipoprotein metabolism and reverse cholesterol transport: strategies for raising HDL cholesterol. Anatol J Cardiol. 2017; 18: 149154.

19. Polimeni L, Del Ben M, Baratta F, Perri L, Albanese F, Pastori D, et al. Oxidative stress: new insights on the association of non-alcoholic fatty liver disease and atherosclerosis. World $\mathrm{J}$ Hepatol. 2015; 7: 1325-1336.

20. Kimoto K, Nakatsuka K, Matsuo N, Yoshioka H. p38 MAPK mediates the expression of type I collagen induced by TGF- $\beta 2$ in human retinal pigment epithelial cells ARPE-19. Invest Ophthalmol Vis Sci. 2004; 45: 2431-2437. 
21. Cote JA, Lessard J, Pelletier M, Marceau S, Lescelleur O, Fradette J, et al. Role of the TGF- $\beta$ pathway in dedifferentiation of human mature adipocytes. FEBS Open Bio. 2017; 7: 10921101.

22. Zhang YE. Non-Smad pathways in TGF- $\beta$ signaling. Cell Res. 2009; 19: 128-139.

23. Collins T, Cybulsky MI. NF-kB: pivotal mediator or innocent bystander in atherogenesis? J Clin Invest. 2001; 107: 255-264.

24. Tawara K, Oxford JT, Jorcyk CL. Clinical significance of interleukin (IL)- 6 in cancer metastasis to bone: potential of anti-IL-6 therapies. Cancer Manag Res. 2011; 3: 177-189.

25. Steinbrenner $H$, Speckmann B, Pinto A, Sies H. High selenium intake and increased diabetes risk: experimental evidence for interplay between selenium and carbohydrate metabolism. J Clin Biochem Nutr. 2011; 48: 40-45.

26. Rayman MP, Stranges S. Epidemiology of selenium and type 2 diabetes: can we make sense of it? Free Radic Biol Med. 2013;65: 1557-1564.

27. Wang XD, Vatamaniuk MZ, Wang SK, Roneker CA, Simmons RA, Lei XG. Molecular mechanisms for hyperinsulinaemia induced by overproduction of selenium-dependent glutathione peroxidase-1 in mice. Diabetologia. 2008; 51: 1515-1524.

28. Misu H, Takamura $T$, Takayama $H$, Hayashi $H$, Matsuzawa-Nagata $N$, Kurita $S$, et al. A liver derived secretory protein, selenoprotein $P$, causes insulin resistance. Cell Metab. 2010; 12: 483-495.

29. Haas JT, Staels B. An oxidative stress paradox: time for a conceptual change? Diabetologia. 2016; 59: 2514-2517.

30. Malaguarnera L, Mededdu R, Palio E, Arena N, Malaguarnera M. Heme oxygenase-1 levels and oxidative stress-related parameters in non-alcoholic fatty liver disease patients. J Hepatol. 2005; 42: 585-591.

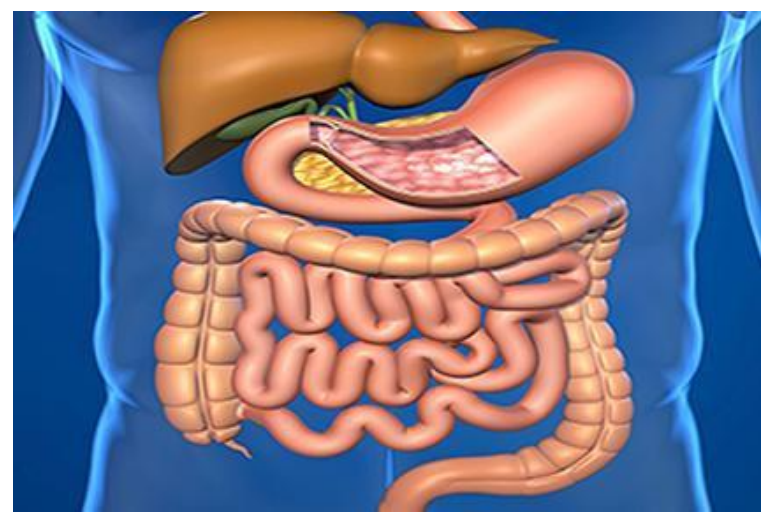

Enjoy OBM Hepatology and Gastroenterology by:

1. Submitting a manuscript

2. Joining in volunteer reviewer bank

3. Joining Editorial Board

4. Guest editing a special issue

For more details, please visit: http://www.lidsen.com/journals/hg 